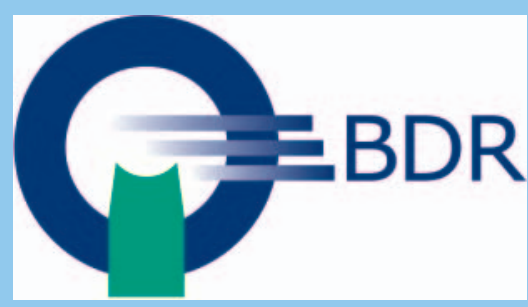

\title{
KBV und Kassen einigen sich zum TSVG - Relevanz für die Radiologie
}

Der Bewertungsausschuss hat Ende Juni beschlossen, dass die im Terminservice- und Versorgungsgesetz (TSVG) vorgesehene extrabudgetäre Vergütung jeweils für eine Arztgruppe und das Behandlungsquartal gilt. Dies betrifft die Terminvermittlung durch die Terminservicestellen (TSS-Terminfall) oder den Hausarzt (s. u. Hausarzt-Vermittlungsfall) ebenso wie die für Radiologen nicht relevanten fünf offenen Sprechstunden und die Behandlung neuer Patienten. Hierbei werden stets alle Untersuchungen und Behandlungen, die Ärzte einer Arztgruppe für einen Patienten im Quartal durchführen (Arztgruppenfall), zu festen Preisen vergütet.

Der Forderung des BDR, insbesondere für die Projektionsradiografie auch für Radiologen die Regelungen zur offenen Sprechstunden anzuwenden, sind KBV und Kassen nicht gefolgt.

Ab September gibt es zusätzlich zeitgestaffelte Zuschläge auf die jeweilige Versicherten-, Grund- oder Konsiliarpauschale (Für Radiologen GOP 24210 - 24212 ; € 4,76 bzw. $€ 5,74)$ von 20,30 und 50 Prozent.

\section{Zuschlag im Termin- servicestellen-Terminfall (TSS-Terminfall) - für Radiologen relevant}

In die Arztgruppenkapitel 3-27 des EBM (ohne Kap. 12 Labor und 19 Pathologie) wird jeweils eine Gebührenordnungsposition (GOP) als Zusatzpauschale für den Zuschlag aufgenommen.

\section{Höhe der Zuschläge}

Die Höhe der Zuschläge ist nach der Länge der Wartezeit auf einen Termin gestaffelt:

- 50 Prozent: Termin innerhalb von acht Tagen sowie in Akutfällen innerhalb von 24 Stunden nach medizinischem Ersteinschätzungsverfahren

- 30 Prozent: Termin innerhalb von neun bis 14 Tagen

- 20 Prozent: Termin innerhalb von 15 bis 35 Tagen

Für die Höhe des Zuschlags ist der Tag der Kontaktaufnahme des Versicherten bei der Terminservicestelle relevant - ab diesem Datum wird gezählt. Dazu werden die TSS den Praxen mit der Übermittlung der Terminbuchung künftig auch dieses Datum mitteilen. Der Arzt kann damit den entsprechend Zuschlag bestimmen und die jeweilige GOP abrechnen. Der Zuschlag ist einmal im Arztgruppenfall berechnungsfähig.

\section{Zuschlag im TSS-Akutfall - für Radiologen relevant}

Einen 50-prozentigen Zuschlag gibt es außerdem für den sogenannten „TSS-Akutfall“ (Termin innerhalb von 24 Stunden nach Ersteinschätzungsverfahren über die 116117).

In die Arztgruppenkapitel 3-27 des EBM (ohne Kap. 12 Labor und 19 Pathologie) wird jeweils eine GOP als Zusatzpauschale aufgenommen, die die Zusatzvergütung für TSSAkutfälle abbildet: ... -Bei Behandlung durch mehrere Arztgruppen werden die Leistungen derjenigen Arztgruppe extrabudgetär vergütet, die den ersten Kontakt zum Patienten im TSVG-Fall hatte. Berechtigt sind alle Arztgruppen die nach den Kapiteln 3-11 und 13 (13.3.1 - 13.3.8), 14 - 18, 20 - 27 sowie nach Abschnitt 30.7. EBM abrechnen.
Offene Sprechstunden (ab 01. September 2019) für Radiologen nicht abrechenbar

Augenärzte, Chirurgen, Frauenärzte, HNOÄrzte, Hautärzte, Kinder- und Jugendpsychiater und -psychotherapeuten, Neurologen, Orthopäden, Psychiater, Urologen

Neupatienten (ab 01. September 2019) - für Radiologen nicht abrechenbar

Eine extrabudgetäre Vergütung für die Behandlung von Neupatienten kommt für alle Arztgruppen zum Tragen, die nach den Kapiteln 3-10, 13 (13.3.1 - 13.3.8), 14, 16, $18,20-23,26-27$ und nach Abschnitt 30.7 EBM abrechnen

Hier sind die Radiologen explizit ausgenommen, nachdem Kapitel 24 EBM nicht umfasst ist.

\section{Hausarzt-Vermittlungsfall}

Haus- sowie Kinder- und Jugendärzte erhalten für die „erfolgreiche Vermittlung“ eines „dringenden“ Facharzttermins ab dem 1. September zehn Euro extrabudgetär. Sie rechnen dafür die neu einzuführende Ziffer ab und geben bei der Abrechnung die Betriebsstättennummer (BSNR) der Praxis an, an die der Patient vermittelt wurde.

Der Zuschlag ist mehrfach berechnungsfähig, wenn der Patient in demselben Quartal durch denselben Arzt zu unterschiedlichen Arztgruppen vermittelt wird. Er ist nicht berechnungsfähig, wenn der vermittelte 
Patient bei demselben Facharzt im laufenden Quartal bereits behandelt wurde.

Der Bewertungsausschuss hat Definitionen festgelegt für:

Dringlichkeit: Ein Termin gilt als dringend, wenn er innerhalb von vier Kalendertagen liegt, nachdem der Hausarzt eine Behandlungsnotwendigkeit festgestellt hat.
Erfolgreiche Vermittlung: Eine erfolgreiche Vermittlung meint die Terminvereinbarung. Konkret heißt das: Der Hausarzt erhält den Zuschlag unabhängig davon, ob der Patient den Termin auch tatsächlich wahrgenommen hat.

Betriebsstättennummer finden: Die BSNR der einzelnen Praxen finden Hausärzte in der Kollegensuche im Sicheren Netz - auch erreichbar über die Telematikinfrastruktur.

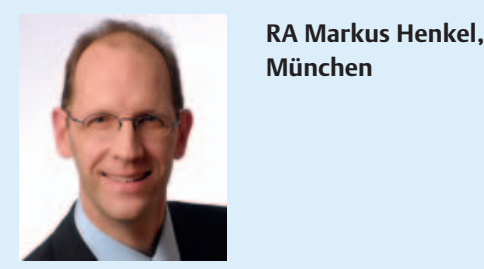

